\title{
Primary malignant bone tumours: Epidemiological data from an Orthopaedic Oncology Unit in South Africa
}

\author{
Dr Y Pillay MBChB, FC Orth(SA) \\ Dr N Ferreira BSc, MBChB, FC Orth(SA), MMed(Orth), PhD \\ Dr LC Marais MBChB, FCS Orth(SA), MMed(Ortho), CIME, PhD \\ Nelson R Mandela School of Medicine, University of KwaZulu-Natal \\ Corresponding author: \\ Dr Yogesh Pillay \\ Tumour, Sepsis and Reconstruction Unit \\ Department of Orthopaedic Surgery \\ Grey's Hospital \\ Nelson R Mandela School of Medicine \\ University of KwaZulu-Natal \\ 3201 Pietermaritzburg, South Africa \\ Tel: +27338973000 \\ Email: yogeshpillay@icloud.com
}

Tumour Sepsis and Reconstruction Unit, Department of Orthopaedic Surgery, Grey's Hospital,

\begin{abstract}
Introduction:

Limited data is available with regard to the epidemiology of primary malignant orthopaedic tumours in the South African clinical setting. As a result, orthopaedic surgeons have to rely on data from other countries when formulating differential diagnoses for malignant bone lesions. Existing data, however, demonstrates variance in the incidence between different geographic regions. By analysing the tumour epidemiology at our centre and comparing it to published data from other parts of the world, we aim to better define the local prevalence of primary malignant bone tumours.
\end{abstract}

Materials and methods:

A retrospective review of all patients with biopsy confirmed malignant primary bone tumours that presented between January 2008 and June 2015 were conducted. Patients with multiple myeloma and lymphoma were excluded. Epidemiological data pertaining to patient demographics, tumour location and histological diagnosis were recorded and analysed.

Results:

Included for review were 117 patients with biopsy-confirmed primary malignant bone tumours. Tumours involving the proximal humerus, distal femur, proximal tibia and pelvis accounted for $80 \%$ of all tumours. Osteosarcoma was the most common histological diagnosis $(72.6 \%)$ and higher than reported figures from any other country. It was followed by chondrosarcoma (11.4\%), Ewing's sarcoma (9.4\%), spindle cell sarcoma (4.2\%) and malignant giant cell tumour (GCT) $(1.7 \%)$. A single patient was diagnosed with adamantinoma. HIV infection had no significant association with primary bone tumour incidence.

\section{Conclusion:}

Epidemiological data from this review reflect small but significant differences compared to international literature. The incidence of osteosarcoma appeared to be higher than in previous reports from other regions. Future study in this area may identify a reason for this difference, socio-economic reasons may be responsible.

Key words: primary bone malignancies, osteosarcoma, epidemiology 


\section{Introduction}

Primary malignant bone tumours are defined as tumours originating in bone. These malignancies are rare, and account for only $0.2 \%$ of all cancers. ${ }^{1}$ By comparison, breast cancer is the most common malignancy in women, accounting for $25 \%$ of all cancers. ${ }^{2,3}$ Despite the rarity, the consequences of malignant primary bone tumours are grave and they are typically associated with high morbidity and mortality. ${ }^{4}$

The scarcity of these tumours combined with the lack of a national database contributes to the lack of South African epidemiological data regarding primary malignant bone tumours. The National Health Laboratory Services has published tumour data based on histological diagnosis. The latest tumour statistics are from 2010 and broadly group all tumours under the heading 'bone'. The lab processed 177 samples during 2010 of which 96 were male and 81 female. Unlike our study, this includes haematological tumours affecting bone including myeloma and lymphoma. ${ }^{5}$ Orthopaedic oncology surgeons in South Africa currently rely on international epidemiological data concerning tumour epidemiology, and statistics from North America and the United Kingdom are frequently referenced. ${ }^{1,6}$

Data from the American Cancer Society suggest that chondrosarcoma is the most common primary malignant bone tumour affecting adults (40\%). Osteosarcoma, chordoma, Ewing's sarcoma and fibrosarcoma follow at $28 \%, 10 \%, 8 \%$ and $4 \%$ respectively. ${ }^{1}$ Reports from the United Kingdom quote osteosarcoma as the most frequently encountered tumour $(34.2 \%)$ followed by chondrosarcoma and Ewing's sarcoma at $27.2 \%$ and $19.3 \%$ respectively. ${ }^{6}$ Epidemiological data from Nigeria report osteosarcoma as their most common malignancy $(58.9 \%)$, followed by chondrosarcoma $(21.4 \%)$, fibrosarcoma $(8.9 \%)$ and Ewing's sarcoma (7.2\%). ${ }^{7}$ The above data clearly demonstrates variance in tumour incidence between geographic regions.

The aim of this study was to determine the relative incidence and describe the demographics of primary malignant bone tumours diagnosed at a South African tumour unit. This retrospective review aims to establish demographic data for primary malignant bone tumours in South Africa.

\section{Materials and methods}

We retrospectively reviewed all patients diagnosed with malignant primary bone tumours at our institution from January 2008 and June 2015. Ethical approval was obtained from our institution's ethics review board prior to commencement of the study. Eligible patients were identified from a prospectively gathered database. Patients were included if they had a biopsy-confirmed diagnosis of primary malignant bone tumour. Patients with multiple myeloma and lymphoma were excluded.
All patients were admitted for local and systemic staging, which was followed by an incisional biopsy. Local staging consisted of radiographs and a magnetic resonance imaging (MRI) scan. Systemic staging included workup for medical co-morbidities, laboratory investigations, computerised tomography (CT) scan and bone scintigraphy. Histology was obtained by formal incisional biopsy in all cases. Diagnosis was subsequently confirmed by combined radiological and histological evaluation. Patient charts were reviewed and data extracted pertaining to patient demographics, tumour location and histological diagnosis.

Statistical analysis was performed using Stata 13.0 (StataCorp. College Station, Texas). Continuous variables were reported as mean $( \pm S D)$ or mean (with interquartile range) and categorical variables as numbers and percentages, unless otherwise stated. Categorical data was compared using the Fisher's exact test. All tests were twosided and the level of significance was set at $\mathrm{p}<0.05$.

\section{Results}

One-hundred-and-seventeen patients met the inclusion criteria. Eighteen patients with multiple myeloma and seven patients with lymphoma were excluded.

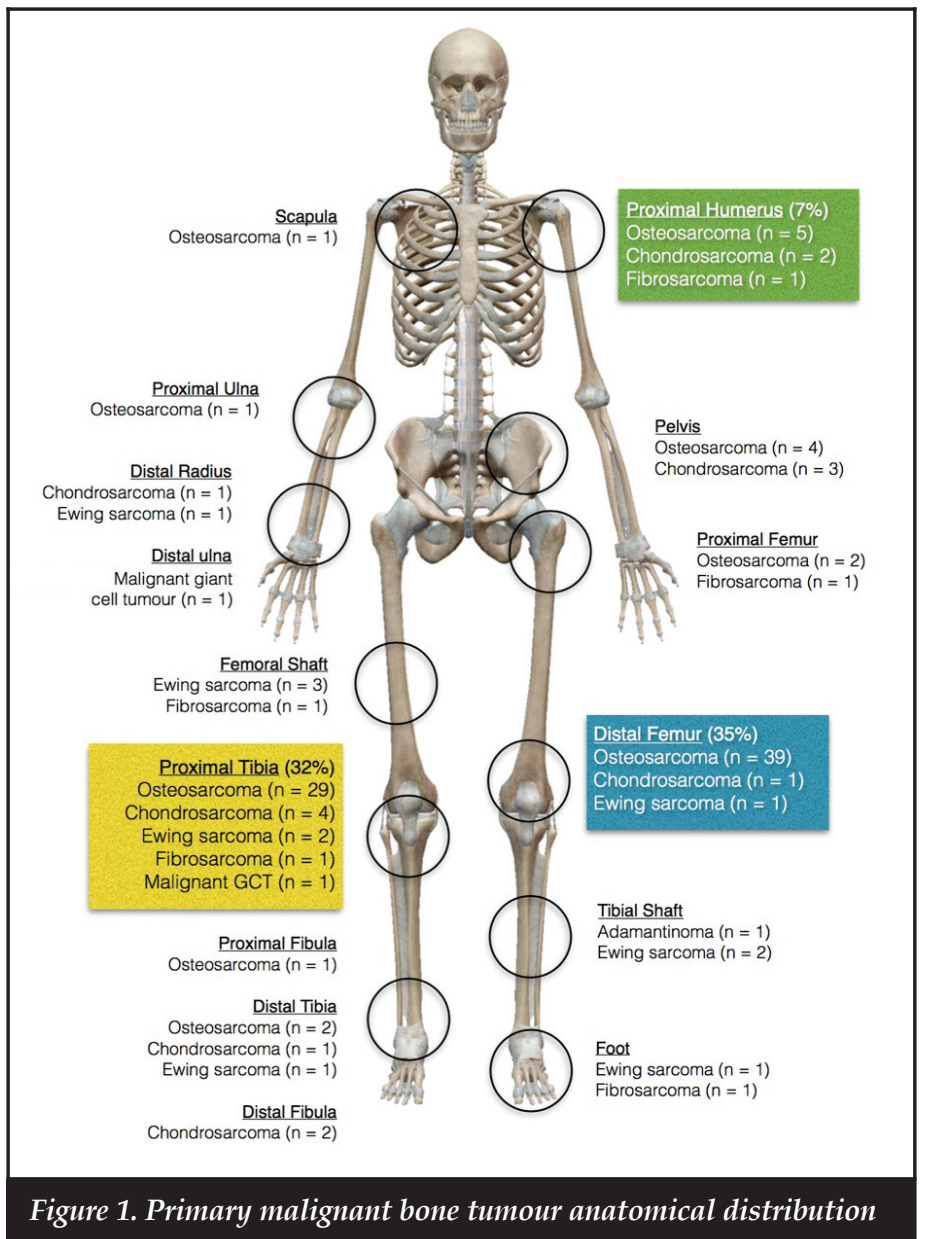


The final cohort consisted of 64 men and 53 women with a mean age of 23.8 years (range 5 to 69). Eighteen patients $(15.4 \%)$ were confirmed to be HIV positive, 95 patients were negative, and the HIV status of four patients was unknown. The majority of tumours (88.9\%) were located in the pelvis and lower limbs, and $80 \%$ of all tumours were confined to four anatomical areas, namely the distal femur, proximal tibia, proximal humerus and pelvis (Figure 1). Osteosarcoma was diagnosed in 85 patients, followed by chondrosarcoma $(\mathrm{n}=13)$, Ewing's sarcoma $(\mathrm{n}=11)$, fibrosarcoma $(\mathrm{n}=5)$, malignant giant cell tumour $(\mathrm{n}=2)$ and adamantinoma $(\mathrm{n}=1)$ (Table I).

\begin{tabular}{|c|c|c|c|c|c|}
\hline & $\begin{array}{l}\text { Grey's } \\
\text { Hospital }\end{array}$ & USA & China & UK & Nigeria \\
\hline Osteosarcoma & $72.6 \%$ & $39.5 \%$ & $59.8 \%$ & $34 \%$ & $58.9 \%$ \\
\hline Chondrosarcoma & $11.1 \%$ & $28.6 \%$ & $16.3 \%$ & $27 \%$ & $21.4 \%$ \\
\hline Ewing's sarcoma & $9.4 \%$ & $11.9 \%$ & $4.4 \%$ & $19 \%$ & $7.2 \%$ \\
\hline Fibrosarcoma & $4.2 \%$ & $5.5 \%$ & $0.8 \%$ & & $8.9 \%$ \\
\hline Chordoma & $0 \%$ & $8.5 \%$ & $4.2 \%$ & & \\
\hline Malignant GCT & $1.7 \%$ & $0.7 \%$ & $1.4 \%$ & & \\
\hline Adamantinoma & $0.9 \%$ & $0.7 \%$ & $0.5 \%$ & & \\
\hline
\end{tabular}

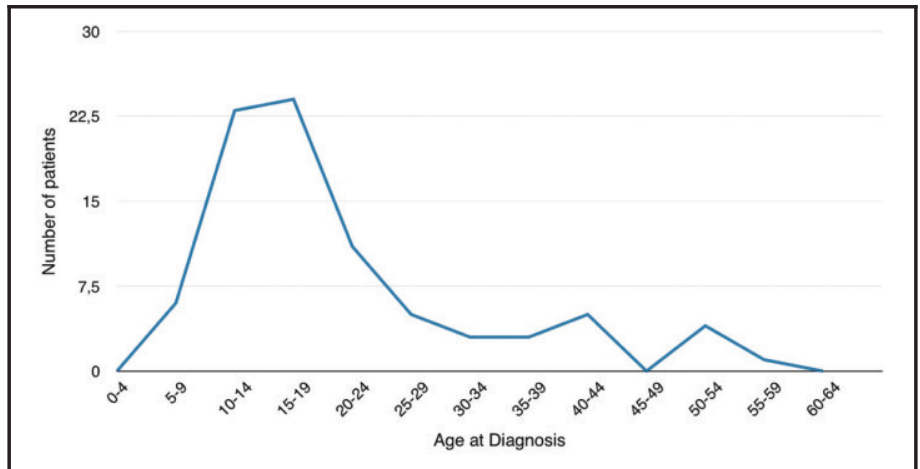

Figure 2. Osteosarcoma age distribution

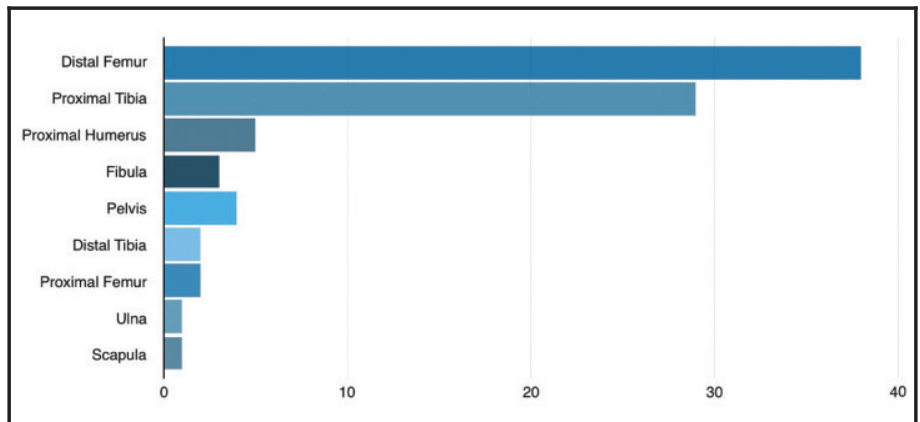

Figure 3. Osteosarcoma anatomical locations

\section{Osteosarcoma}

Osteosarcoma was the most common primary malignant bone tumour and accounted for $72.6 \%$ of all cases. There was a slight male predominance with a male-to-female ratio of 1.1:1. Mean age at diagnosis was 21 years (range 6-56). The incidence failed to show a bimodal age distribution and only had a single peak during adolescence (15-19 years) (Figure 2). The majority of osteosarcomas $(78.8 \%)$ arose around the knee with 38 out of $85(44.7 \%)$ involving the distal femur and 29 out of $85(34.2 \%$ ) involving the proximal tibia (Figure 3).

Histological variants consisted of $73(85.8 \%)$ high grade intra-medullary (conventional), four periosteal $(4.7 \%)$, four telangiectatic $(4.7 \%)$, three high grade surface (3.9\%) and one parosteal $(1.4 \%)$ osteosarcoma (Table II). Conventional osteosarcomas were histologically further subdivided into $55(75.3 \%)$ osteoblastic, $15(20.5 \%)$ chondroblastic and three (4.1\%) fibroblastic variants.

Juxta-cortical lesions were the most common osteosarcoma variant. Periosteal osteosarcomas were diagnosed in two women and two men and accounted for $4.7 \%$ of all osteosarcomas. Median age at presentation was 18 years (range 830). All four tumours arose from the proximal tibia. High grade surface lesions were diagnosed in three patients $(3.9 \%)$. All three patients were women with a median age of 33 years (range 23-40). Two patients were HIV positive. All three tumours were located around the knee with two arising from the distal femur and one from the proximal tibia. A single $(1.4 \%)$ parosteal osteosarcoma was diagnosed in the distal femur of a 24-year-old HIV-positive man.

Telangiectatic osteosarcoma was seen in four $(4.7 \%)$ patients. These included two men and two women. Median age was 26 years (range 14-40). Two lesions were located in the distal femur and two in the proximal humerus.

Our data failed to show any significant association between HIV infection and conventional osteosarcoma ( $p=0.268)$, juxta-cortical lesions ( $\mathrm{p}=0.086$ ), high grade surface lesions ( $\mathrm{p}=0.342)$ or telangiectatic osteosarcoma $(\mathrm{p}=0.068)$. When patients whose HIV status was not known were excluded, however, a significant association between HIV infection and high-grade surface osteosarcomas was observed $(\mathrm{p}=0.050)$.

\begin{tabular}{|c|c|c|c|}
\hline & $\begin{array}{c}\text { Grey's } \\
\text { Hospital }\end{array}$ & USA & China \\
\hline Conventional osteosarcoma & $86.8 \%$ & $78.4 \%$ & $90 \%$ \\
\hline Telangiectatic osteosarcoma & $3.9 \%$ & $3.6 \%$ & $1.1 \%$ \\
\hline Small cell osteosarcoma & $0 \%$ & NA & $0.9 \%$ \\
\hline Low-grade central osteosarcoma & $0 \%$ & $1.1 \%$ & $0.7 \%$ \\
\hline Secondary osteosarcoma & $0 \%$ & $10.3 \%$ & $0.6 \%$ \\
\hline Parosteal osteosarcoma & $1.4 \%$ & $4.1 \%$ & $5.1 \%$ \\
\hline Periosteal osteosarcoma & $3.9 \%$ & $1.7 \%$ & $0.9 \%$ \\
\hline High-grade surface osteosarcoma & $3.9 \%$ & $0.7 \%$ & $0.8 \%$ \\
\hline
\end{tabular}




\section{Chondrosarcoma}

Thirteen patients $(11.1 \%)$ were diagnosed with chondrosarcoma. These tumours were more common in men with a male-to-female ratio of 1.6:1 and exhibited a trend of increased incidence with advancing age (mean 39.2 years, range 15-65) (Figure 4). The most common location was the lower limb (tibia, femur, fibula), accounting for $53.8 \%$ of cases followed by three tumours in the upper limb (humerus, radius) and three in the pelvis (Figure 5). The majority of chrondrosarcomas $(84.6 \%)$ were classified as low and intermediate grade (grade I and II), with only two cases $(15.4 \%$ ) being classified as high grade (grade III).

\section{Ewing's sarcoma}

Ewing's sarcoma was diagnosed in 11 patients $(9.4 \%)$. These tumours occurred exclusively during the first two decades of life with a mean age of 13.2 years (range 5-20) at time of diagnosis (Figure 6). There was a male predominance with a male-to-female ratio of 1.75:1. The most common primary site was the lower limb (90.1\%), with the tibia being involved in five cases, femur in four cases and foot in one case. The remaining case involved the radius (Figure 7).

\section{Spindle cell sarcoma}

Spindle cell sarcoma was diagnosed in five patients (4.2\%). This group was composed of three fibrosarcomas and two undifferentiated sarcomas of bone. Three men and two women with a median age of 46 years (range 9-63) were affected. The lower limb (tibia, femur, foot) was most commonly involved $(80 \%)$ followed by a single occurrence in the humerus.

\section{Malignant giant cell tumour of bone (GCT)}

Malignant GCT of bone was diagnosed in two patients $(1.7 \%)$. These tumours occurred in a 36-year-old man and 63 -year-old women and involved the proximal tibia and distal ulna respectively.

\section{Adamantinoma}

A single patient was diagnosed with an adamantinoma. This tumour was located in the midshaft of the tibia of a 34-year-old HIV-positive woman.

Our data failed to show any significant association between HIV infection and the incidence of any primary malignant bone tumours $(\mathrm{p}=0.698)$.

\section{Discussion}

This retrospective review represents novel demographic data for primary malignant bone tumours from a developing region in South Africa. While overall tumour statistics grossly mirror international data, significant differences were observed. Osteosarcoma was the most frequently diagnosed tumour, in keeping with international statistics.
The relative incidence of osteosarcoma compared to other primary malignant bone tumours, however, was higher than in previous reports. Osteosarcoma made up $73 \%$ of all tumours compared to $40 \%, 34 \%, 60 \%$ and $59 \%$ in the US, UK, China and Nigeria respectively. ${ }^{8,9}$ The incidence of osteosarcoma in our cohort was more than double that of the UK. It is notable that the incidence of osteosarcoma is higher in developing countries than their developed counterparts.

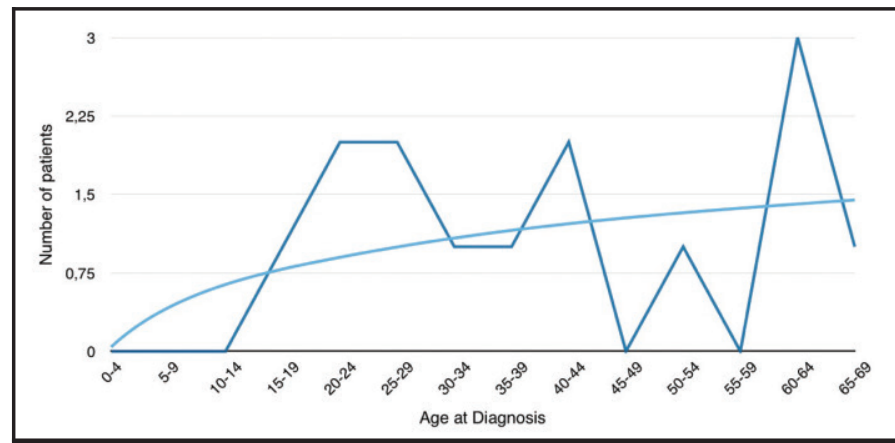

Figure 4. Chondrosarcoma age distribution

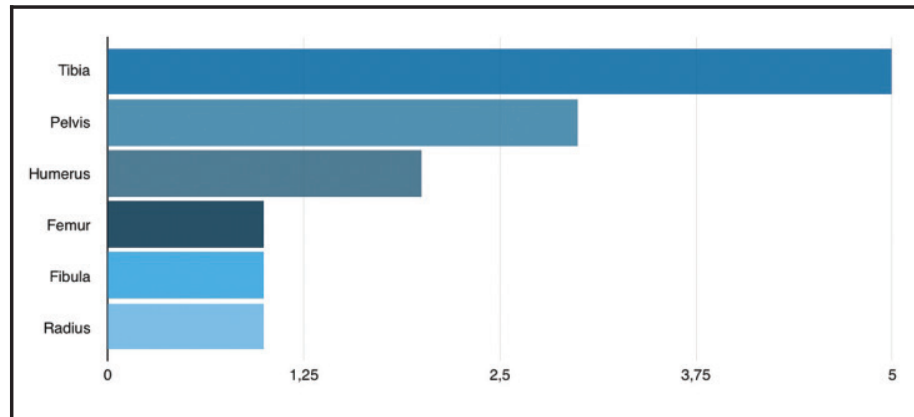

Figure 5. Chondrosarcoma anatomical locations

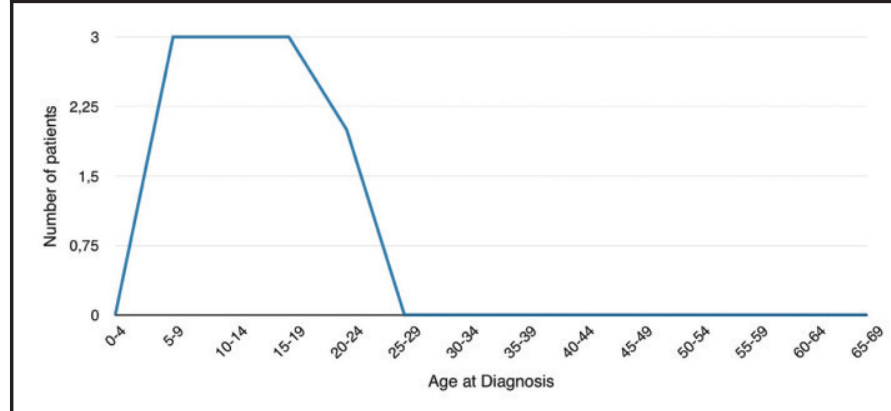

Figure 6. Ewing's sarcoma age distribution

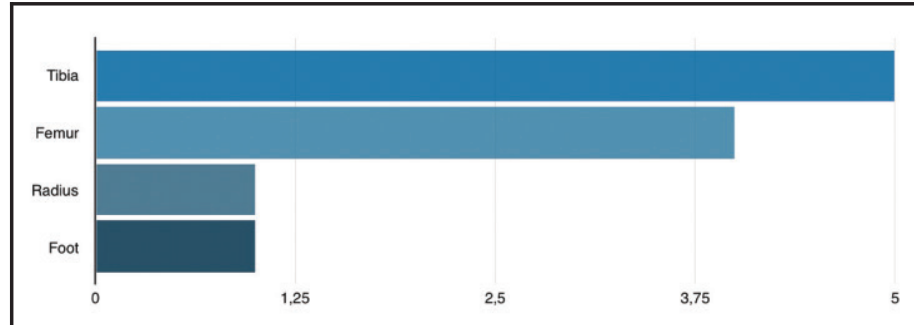

Figure 7. Ewing's sarcoma anatomical locations 
A possible explanation for this discrepancy is the younger average age of the population in those countries. The average age of the populations in Nigeria and South Africa are 18 years and 25 years respectively, compared to 36 years and 40 years in the US and UK. ${ }^{10} \mathrm{~A}$ higher incidence of HIV infection in South Africa compared to these other countries might also influence the incidence of osteosarcoma but further research into this association is required. ${ }^{11}$ The lower average population age could also explain why our data failed to show a bimodal age distribution, with only a single peak identified in adolescence. ${ }^{12}$ This difference in relative incidence is worth further investigation. If the difference cannot be explained on the basis of population age difference alone, it could imply that certain socio-economic factors contribute to the development of osteosarcoma.

Chondrosarcoma represented the second most common tumour at our institution. This tendency was also reflected in the literature from the US, UK, China and Nigeria. The incidence in our cohort, however, was significantly lower than in other geographic regions, with chondrosarcoma accounting for $29 \%, 27 \%, 16 \%$ and $21 \%$ of primary malignant bone tumours in the US, UK, China and Nigeria ${ }^{9}$ respectively. The discrepancy in observed incidence may again be a reflection of the relative age differences of the reported populations as chondrosarcoma typically affect older patients. A trend of increased incidence of chondrosarcoma with advancing age was also demonstrated in our data. International literature reports a high incidence of chondrosarcoma located in the pelvis; however, in our study this was less frequent than in the tibia.

The incidence of Ewing's sarcoma varies significantly in published literature. The highest incidence was reported from the UK (19\%), compared to only $4 \%$ from China. Our results fall within this range and accounted for $9 \%$ of all primary malignant bone tumours diagnosed. Ewing's sarcoma's predilection for the long bones of the lower limb was mirrored in this series.

Tumour location also differed from published data. The top anatomical locations in our data was the distal femur $(33 \%)$, proximal tibia $(24 \%)$, humerus $(7 \%)$ and pelvis $(6 \%)$. By comparison, data from the Mayo Clinic and JST Hospital (China) revealed the distal femur (25\% and $42.3 \%)$, tibia $(9.6 \%$ and $19.6 \%)$ and pelvis (14\% and $11 \%)$ as the top three anatomical locations.

Several limitations are identified in our study including its retrospective design and single centre cohort. The vast majority of our patients were of African descent and this might skew the findings. This study presents novel data from South Africa but the sample size is relatively small compared to similar series from the US, Europe and China. Although the associations between HIV infection and sarcomas were reported, this study was not specifically designed to identify these associations. We hope that this research stimulates other similar studies elsewhere in the country, eventually yielding a sample size as large as other centres.

\section{Conclusion}

Differences in the relative incidence of certain malignant primary bone tumours, in comparison with European, American, Nigerian and Chinese populations, were identified. An especially high incidence of osteosarcoma and correspondingly low incidence of chondrosarcoma was noted in our patient cohort. More research into the possible reasons for the apparent increase in the relative incidence of osteosarcoma is warranted.

\section{Compliance with ethics guidelines}

Ethical approval was obtained from our institution's Biomedical Research Ethics Committee prior to commencement of the study.

Drs Pillay, Ferreira and Marais declare that the content of this article is their original work. No benefits of any form have or will be received from any commercial party related directly or indirectly to the subject of this article.

\section{References}

1. American cancer society [internet]. Available from: http:// www.cancer.org/cancer/bonecancer/detailedguid/bone-cancerkey-statistics

2. Jensen A, Jacobsen JB, Norgaard M, Yong M, Fryzek JP, Sorensen HT. Incidence of bone metastases and skeletal-related events in breast cancer patients: A population-based cohort study in Denmark. BMC Cancer. 2011;11:29.

3. Wcrf.org. World Cancer Research Fund International [Internet]. 2016 [cited 19 January 2016]. Available from: http:/ / www.wcrf.org.

4. Bramer JAM, Somford MP. The epidemiology of primary skeletal malignancy. Orthopaedics and trauma 2010;24(4);247-51.

5. National Health Laboratory Service. Tumours diagnosed by histology [Internet]. 2010 [cited 19 January 2016]. Available from: http: / / www.nioh.ac.za / assets / files / NCR_Final_2010_tables(1).pdf

6. Arora RS, Alston RD, Eden TOB, Geraci M, Birch JM. The contrasting age-incidence patterns of bone tumours in teenagers and young adults: Implications for aetiology. Int. J. Cancer. 2012;131:1678-85. doi:10.1002/ijc. 27402.

7. Daw NC, Billups CA, Rodriguez-Galindo C, McCarville MB, Rao BN, Cain AM, Jenkins JJ, Neel MD, Meyer WH. Metastatic osteosarcoma. Cancer. 2006;106:403-12. doi: 10.1002/ cncr.21626

8. Obalam DC, Giwa SO, Banjo AF, Akinsulire AT. Primary bone tumours in a tertiary hospital in Nigeria: 25-year review. Niger J Clin Pract. 2009;12(2)169-72.

9. Niu X, Xu H, Inwards C, Li Y, Ding Y, Letson G et al. Primary bone tumors: epidemiologic comparison of 9200 patients treated at Beijing Ji Shui Tan Hospital, Beijing, China, with 10165 patients at Mayo Clinic, Rochester, Minnesota. Archives of Pathology \& Laboratory Medicine. 2015;139(9):1149-55.

10. Cia.gov. The World Factbook [Internet]. 2016 [cited 19 January 2016]. Available from: https://www.cia.gov/library/publications/ resources/the-world-factbook/

11. Marais LC, Ferreira N. Osteosarcoma in patients living with HIV/AIDS. ISRN Oncology. 2013;1-6. doi:10.1155/ 2013/219369.

12. Ottaviani G, Jaffe N. The epidemiology of osteosarcoma. Cancer Treat Res. 2009;152:3-13. doi:10.1007/ 978-1-4419-0284-9_1.

This article is also available online on the SAOA website (www.saoa.org.za) and the SciELO website (www.scielo.org.za). Follow the directions on the Contents page of this journal to access it. 